\title{
COVID-19 and other Respiratory Conditions - A Narrative Review of the Impact of the COVID-19 Pandemic on other Respiratory Conditions and Modifications Made to Adult Respiratory Services in the United Kingdom
}

\author{
Rabiu Momoh ${ }^{1 *}$ \\ ${ }^{1}$ Maidstone Hospital, Maidstone, Kent, United Kingdom
}

The COVID-19 pandemic that started from the Wuhan area of China has continued to receive significant worldwide attention due to its severe morbidity and increasing mortality figures [1]. The effect of the COVID-19 virus infection on different organ systems in the human body has been described in the literature (COVID-associated encephalitis, nephritis, pancreatitis, lymphopenia, thromboembolic events, and gastro-enteritis among others) [2]. The most debilitating effect of the COVID-19 virus on the human body system is its effect on the respiratory system, causing an acute respiratory distress syndrome (with manifestations from mild to severe). Most mortalities from the COVID-19 viral infection are largely a consequence of severe acute respiratory distress syndrome (ARDS) associated with this virus [3].

In this article, we have explored the impact of the COVID-19 pandemic on those with pre-existing respiratory conditions, as well as exploring modifications required to respiratory services in the United Kingdom to cope with the challenges posed by the COVID-19 virus.

As of $17^{\text {th }}$ January 2021, there have been about 3.36 million cases of COVID-19 infection recorded in the United Kingdom, with about 88,590 COVID-19-related deaths recorded [4]. The first wave of the COVID-19 spread in the United Kingdom (between late February and mid-July 2020) was accompanied by significant disruption to respiratory services, and changes were made. These services that included various kinds of clinics (non-invasive ventilation (NIV) clinics, tuberculosis clinics, and regular chest clinic sessions) were interrupted, and efforts were made to explore virtual (telephone and video) consultations. Routine respiratory investigations that required aerosol generation (lung function tests, endoscopic studies) were also suspended till service modifications to ensure safety to hospital users (staff members and patients alike) were largely guaranteed.

The United Kingdom has an estimated 1.2 million people living with chronic obstructive pulmonary disease (COPD) [5]. There are about 5.4 million persons in the UK living with asthma (among whom are about 1 million persons with 'difficult' asthma and a further 200,000 sub-set with severe asthma) [6]. According to the British Lung Foundation, about 85000 persons in the UK are with a lung cancer diagnosis [7]. Also, about 10,600 persons are known to have a diagnosis of cystic fibrosis in the UK [8]. The significant impact of the COVID-19 virus and lockdown measures on routine services to these vulnerable groups of individuals are continually being seen and measures made to ease them.
In the pre-COVID era, it is known that COPD patients usually are at risk of viral exacerbation of their condition [9]. Existing studies suggest that the increased expression of the ACE receptor, which serves as the portal of entry for the COVID-19 virus, may predispose COPD patients to be susceptible to contracting the COVID-19 virus [10]. Patients with severe COPD are classed as high risk or clinically extremely vulnerable to COVID-19 infection in the UK, and those with moderate COPD as being of moderate risk [11]. Variable figures about the impact of the COVID-19 pandemic on COPD patients across different countries may be largely put down to the effect of shielding measures being used by this group of persons. It is, however, largely known that the impact of the COVID-19 virus infection is largely more severe in patients with COPD [12].

Literature evidence for increased risk of contracting or severity of COVID-19 in asthmatics varies in their assertions about these clinical entities. The Centre for Disease Control and Prevention states that patients with moderate or severe asthma are at risk of severe manifestations of COVID-19 infection [13]. The United Kingdom government class individuals with severe asthma as being at higher risk from COVID-19 severity, and patients with severe asthma and those with other chronic respiratory conditions such as COPD, cystic fibrosis, are rated in the priority group to receive COVID-19 vaccination [14]. Lieberman-Cribbin, et al. in their publication (December 2020) assessing mortality in COVID-19 patients from the Mount Sinai Health Systems COVID-19 Registry in the United States of America, with asthma as a co-morbidity, revealed that there was no statistical significance between asthma and mortality in asthma patients with COVID-19 ( $p$-value 0.506) and patients without COVID-19 (p-value 0.817) on a univariate analysis [15].

*Corresponding author: Rabiu Momoh, Maidstone Hospital, Maidstone, Kent, United Kingdom

Accepted: July 21, 2021

Published online: July 23, 2021

Citation: Momoh R (2021) COVID-19 and other Respiratory Conditions - A Narrative Review of the Impact of the COVID-19 Pandemic on other Respiratory Conditions and Modifications Made to Adult Respiratory Services in the United Kingdom. Ann Pulmonol 5(1):56-59 
The potential dangers of hospital visits or admissions for patients with chronic respiratory conditions (e.g., COPD, cystic fibrosis, severe asthma, advanced lung cancer) as it was noted that hospital centres potentially became vectors for COVID-19 infection to this group of patients and others despite measures taken to reduce crossinfection in hospital environments [16]. Modalities taken to reduce this potential risk were to adopt as many virtual medical reviews (using telephone or video calls) for these patients and, where needed, home visits. Where the clinical indications are worth it and the risk of hospital visits well-considered, hospital contacts were then recommended.

There were potential concerns for community respiratory services that supported patients with chronic respiratory conditions requiring home non-invasive ventilation (NIV) or long-term oxygen therapy. The concerns were largely from the point of view of aerosolgeneration from NIV use and the risk of transmission of the COVID-19 virus to carers or family members from potentially undiagnosed patients. There were also potential concerns that the visiting service providers (Community respiratory team) could potentially be modes of transmission of the COVID-19 infection to these at-risk patients, who were largely recommended for shielding as a means of preventing COVID-19 virus contraction [17].

The unique differences in the ARDS caused by the COVID-19 virus compared to ARDS due to other causes known in the pre-COVID era have been brought up in literature. A significant disruption of lung perfusion mechanics, loss of protective hypoxic pulmonary vasoconstriction, increased pulmonary vascular thrombosis, reninangiotensin-aldosterone system disruption has been described in COVID-19-induced ARDS, causing severe hypoxemia [18]. The implication of this for patients with underlying chronic respiratory conditions could mean a worse severity due to their underlying lung or lung function compromise.

As experienced in many hospital centres, there had to be a delineation of hospital areas into COVID, likely or suspected COVID and non-COVID areas, and patients' flow in the hospitals are determined by their COVID-status and individualized hospital needs [19]. The early period of the first wave of the COVID-19 pandemic in the UK witnessed the challenge of turn-around times for results of COVID-19 swabs obtained from patients as most presentations for COVID-like symptoms (fever, cough, shortness of breath) could also be due to other respiratory conditions such as asthma, COPD exacerbation, community-acquired pneumonia, complicated influenza, interstitial lung disease flare, pulmonary embolism among other conditions [20].

Efforts at managing the scourge of this pandemic in the UK emphasized many significant aspects of the respiratory specialty. Respiratory specialists were at the lead for non-invasive ventilation options for patients with COVID-19 pneumonitis (or the ensuing ARDS). They have been involved in management decision making about oxygen supply and pressure delivery systems in hospitals. Blood gas analysis services were further enhanced during this pandemic. Respiratory specialists have jointly helped to coordinate efforts with hospital managements, microbiologists, infection control units, and critical care service leads [21].The concepts around the management of COVID-19 pneumonitis (and the ensuing ARDS) became a knowledge skill for a wide range of medical professionals (including those deployed from other service areas to join efforts at managing COVID-19 patients) had to improve knowledge [22].

Advanced care plans and treatment escalation protocols for patients have become a key plan at admission points for COVID-19 patients arriving through the respiratory emergency department of many hospitals. Patients are reviewed holistically, putting their co-morbidities, performance status, and patients' wishes into perspective at determining what should make for escalated treatment plans in hospitals [23].

Knowledge and guidelines for the safe management of patients with COVID-19 pneumonitis and COVID-19-induced ARDS have continued to evolve with improving knowledge about this severe disease. The first COVID-19 wave in the UK was approached with early endotracheal intubation measures and admission into the intensive care units where indicated. The ongoing second wave of COVID-19 infection in the UK has been approached differently, with considerations given to delayed endotracheal intubation while trying out non-invasive ventilation modes on dedicated respiratory high dependency unit areas in the early phases preceding escalation into the intensive care unit for mechanical ventilation (if needed) [24].

Different treatments that are either part of ongoing trials or products of concluded trials are a large part of treatment protocols have become a feature in the care of patients with COVID-19 pneumonitis or ARDS. Steroids (e.g., dexamethasone), aspirin, clarithromycin, remdesivir, synthetic monoclonal antibodies, plasma antibody therapy, and interleukin- 6 inhibitors (tocilizumab, sarilumab) are being deployed in many UK centres as needed. Extracorporeal membrane oxygenation, as part of a coordinated national program, are also in use where indicated [25].

As of the week ending on the $24^{\text {th }}$ of January $2021,6,232,584$ COVID-19 vaccinations have been done in the United Kingdom to $5,792,159$ individuals. Forty-two percent of these figures being administered to the UK population group above 80 years old [26]. It is hoped that the COVID-19 vaccine would help reduce the burden of the COVID-19 virus in the UK as vaccine coverage continues to increase.

Expanded rehabilitation services for individuals with the longterm sequelae of severe COVID-19 infection, an entity described as "Long COVID" or "Post-COVID Syndrome," have been seen, and regional care plans for this unique group of persons is ensured. Pulmonary, physical, psychological, occupational, and sometimes cardiac rehabilitation needs are being recorded in this cohort of persons. Latest data within the United Kingdom estimates about 60,000 persons to be living with 'Long COVID,' and there are currently 69 initial assessment service centres scattered across the country earmarked to identify unique needs of persons with PostCOVID syndrome and to coordinate their ongoing care [27].

Annual influenza jabs in the United Kingdom did continue ahead of the winter seasons of the year 2020, especially among healthcare workers. There was a unique challenge reported by General Practitioners in a DAUK study, with $42 \%$ out of 900 respondents noting challenges with vaccinating people in the winter season of 2020. Eight percent of GP respondents reported some patients were unwilling to visit GP centers to obtain their jabs. Modifications like drive-through were considered as corrective measures in certain areas [28].

The public health measures (shielding for vulnerable adults, encouraging face mask usage, encouraging hand sanitization, social distancing, restriction of travels, flu, and COVID vaccination efforts) encouraged during the ongoing period of the COVID-19 pandemic in the UK is helping to control not just the impact of the COVID-19 pandemic, but also helping to keep the impact of COVID-19 and influenza virus co-infection under check. Dr. Soo J, et al. in their August 2020 publication did highlight the significant reduction in influenza-like illnesses due to public health measures deployed in Singapore at controlling the COVID-19 virus and agreed these would help reduce the impact of the virus in their country [29].

A case series of 6 co-infections with COVID-19 and influenza virus was described by Resat O, et al. in June 2020 , and they recommended 
Citation: Momoh R (2021) COVID-19 and other Respiratory Conditions - A Narrative Review of the Impact of the COVID-19 Pandemic on other Respiratory Conditions and Modifications Made to Adult Respiratory Services in the United Kingdom. Ann Pulmonol 5(1):56-59

that expanded screening for other respiratory pathogens (e.g., Legionella, cytomegalovirus, Mycoplasma, rhinovirus, parainfluenza among others) should be considered during the hospital care of COVID-19 cases. They stated in their case series that patients with COVID-19 and influenza co-infection were at a higher risk of superimposed bacterial pneumonia [30].

In a nationwide comparative retrospective study of hospital admissions (for 89530 COVID-19 cases and 45819 seasonal influenza cases) done in France by Lionel $\mathrm{P}$, et al. they found that COVID-19 was associated with worse pathogenicity than seasonal influenza in patients with fewer co-morbidities. They also stated that patients admitted with COVID were more likely to be obese or overweight, have diabetes mellitus, hypertension, or dyslipidemia, while patients hospitalized for seasonal influenza were more likely to have underlying COPD, liver cirrhosis, heart failure, and deficiency anaemia [31].

Pulmonary embolism complicating COVID-19 infection has been described, and thromboprophylaxis should be considered for hospitalized patients with COVID-19 infection. The mechanisms of these blood clots have been attributed to blood vessel dysfunctions and procoagulant states set off by the inflammatory process attributed to the COVID-19 infection, as evident by raised D-dimer values in COVID-19 patients with pulmonary embolism. Long stay required on ventilatory support for those admitted into intensive care units raise the problem of immobility as an adjoining cause of increased clotting situation in COVID-19 patients [32].

Sakr Y, et al. in their Sept 2020 publication, did quote an estimated 2.6-8.9\% of hospitalized COVID patients developing pulmonary embolism, and a third of those requiring intensive care unit admission for COVID-19 would develop a pulmonary embolism [33]. Saquib S, et al. reported a case of a 36-year-old female with COVID-19, who became symptomatic for bilateral extensive pulmonary embolism and required thrombolysis. The patient experienced resolution of symptoms (chest pain and shortness of breath) post-thrombolysis and was discharged on novel oral anticoagulants [34].

Globally, there is increasing knowledge about the COVID-19 virus, and intersectoral collaboration is being adopted to overcome the challenges it is still posing. Vaccines against the COVID-19 virus have been touted as an instrument of hope in worst-hit areas, among other measures. The gain in knowledge that has been achieved in the field of respiratory medicine, working closely with other units (like infection control, microbiology, critical care, public health specialists, and hospital administration teams) from the ongoing COVID-19 pandemic will chart away into the future to help tackle any further similar unforeseen event(s).

\section{Conflicts of Interest}

None to declare.

\section{Funding}

None required.

\section{References}

1. Guo YR, Cao QD, Hong ZS, et al. (2020) The origin, transmission, and clinical therapies on coronavirus disease 2019 (COVID-19) outbreak - an update on the status. Mil Med Res 7: 11.

2. Gavriatopoulou M, Korompoki E, Fotiou D, et al. (2020) Organspecific manifestations of COVID-19 infection. Clin Exp Med 20: 493-506.

3. Riadh B, Mousa MA, Michèle VE, et al. (2020) Acute respiratory distress syndrome: A life-threatening associated complication of SARSCoV-2 infection inducing COVID-19. J Biomol Struct Dyn 5: 1-10.
4. NHS (2021) COVID-19 daily deaths.

5. British Lung Foundation (2021) Chronic obstructive pulmonary disease (COPD) statistics.

6. Asthma UK (2021) Asthma facts and statistics.

7. British Lung Foundation (2021) Lung cancer statistics.

8. Cystic Fibrosis Trust. What is Cystic Fibrosis?

9. Hammad Q, Amir S, Nicola AH (2014) Chronic obstructive pulmonary disease exacerbations: Latest evidence and clinical implications. Ther Adv Chronic Dis 5: 212-227.

10. Janice ML, Masahiro N, Cheng W, et al. (2020) COVID-19 and COPD. Eur Respir J 56: 2002108.

11. NHS (2021) Who's at higher risk from coronavirus.

12. Amy AA, Joe Z, Umur SH (2020) SARS-CoV-2 infection in the COPD population is associated with increased healthcare utilization: An analysis of Cleveland clinic's COVID-19 registry. EClinical Medicine 26: 100515

13. CDC (2021) COVID-19.

14. GOV.UK (2020) Joint Committee on Vaccination and Immunisation: Advice on priority groups for COVID-19 vaccination.

15. Lieberman-Cribbin W, Rapp J, Alpert N, et al. (2020) The impact of asthma on mortality in patients with COVID-19. Chest 158: 2290-2291.

16. Gareth I (2020) Covid-19: Doctors sound alarm over hospital transmissions. BMJ 369.

17. Jillian GB, Milind S (2020) Case for continuing community NIV and CPAP during the COVID-19 epidemic. Thorax 75: 368.

18. Harry K, Rajarajan A, Steven P, et al. (2020) Emerging mechanisms of pulmonary vasoconstriction in SARS-CoV-2-induced acute respiratory distress syndrome (ARDS) and potential therapeutic targets. Int J Mol Sci 21: 8081.

19. Morton W (2020) Clinicians are leading service reconfiguration to cope with covid-19. BMJ 369: 1444.

20. Coleman J, Kaveh M, Ella JM, et al. (2020) COVID-19: To be or not to be-that is the diagnostic question. Postgrad Med J 96: 392-398.

21. Jacqui $T$ (2020) Clinicians are leading service reconfiguration to cope with covid-19. BMJ 369: m1444.

22. Thomas WN, Nick PT, Annabel N, et al. (2020) Respiratory failure and non-invasive respiratory support during the covid-19 pandemic: An update for re-deployed hospital doctors and primary care physicians. BMJ 369: m2446.

23. Luke M (2020) Can the COVID-19 crisis strengthen our treatment escalation planning and resuscitation decision making? Age Ageing 49: 525.

24. Ingrid T (2021) Covid-19: When to start invasive ventilation is "the million-dollar question". BMJ 372: n121.

25. Luigi C, Christopher M, Stephane L, et al. (2021) Consensus on the referral and admission of patients with severe respiratory failure to the NHS ECMO service. Lancet Respir Med 9: e16-e17.

26. NHS (2021) COVID-19 vaccination statistics.

27. NHS (2020) Post-COVID Ssndrome (Long COVID).

28. Awil M (2020) Three-quarters of GPs struggling to get enough flu vaccines, MPs warned. Pulse. 
Citation: Momoh R (2021) COVID-19 and other Respiratory Conditions - A Narrative Review of the Impact of the COVID-19 Pandemic on other Respiratory Conditions and Modifications Made to Adult Respiratory Services in the United Kingdom. Ann Pulmonol 5(1):56-59

29. Soo JRJ, Calvin JC, Stefan M, et al. (2020) Decreased influenza incidence under COVID-19 Control Measures, Singapore. Emerg Infect Dis 26: 1933-1935.

30. Resat O, Rasim C, Arif D, et al. (2020) Influenza and COVID-19 coinfection: Report of six cases and review of the literature. J Med Virol 92: 2657-2665.

31. Lionel P, Jonathan C, Anne-Sophie M, et al. (2020) Comparison of the characteristics, morbidity, and mortality of COVID-19 and seasonal influenza: A nationwide, population-based retrospective cohort study. The Lancet 9: 251-259.
32. Wikipedia contributors (2021) COVID-19. In: Wikipedia, The Free Encyclopedia.

33. Sakr Y, Giovini M, Leone M, et al. (2020) Pulmonary embolism in patients with coronavirus disease-2019 (COVID-19) pneumonia: A narrative review. Ann Intensive Care 10: 124.

34. Saquib NS, Sonam T (2020) A case of acute massive pulmonary embolism in Covid19 pneumonitis. IOSR Journal of Dental and Medical Sciences 19: 19-22. 